LAWRENCE LIVERMORE N A T IO N A L LABORATORY

Effects of Initial Conditions on

Compressible Mixing in

Supernova-Relevant Laboratory

Experiments

A. R. Miles, M.J. Edwards, J.A. Greenough

May 3, 2004

High Energy Density Laboratory Astrophysics (HEDLA) 04

Tucson, AZ, United States

March 10, 2004 through March 13, 2004 
This document was prepared as an account of work sponsored by an agency of the United States Government. Neither the United States Government nor the University of California nor any of their employees, makes any warranty, express or implied, or assumes any legal liability or responsibility for the accuracy, completeness, or usefulness of any information, apparatus, product, or process disclosed, or represents that its use would not infringe privately owned rights. Reference herein to any specific commercial product, process, or service by trade name, trademark, manufacturer, or otherwise, does not necessarily constitute or imply its endorsement, recommendation, or favoring by the United States Government or the University of California. The views and opinions of authors expressed herein do not necessarily state or reflect those of the United States Government or the University of California, and shall not be used for advertising or product endorsement purposes. 
UCRL-PROC-202919

\title{
Effects of Initial Conditions on Compressible Mixing in Supernova-Relevant Laboratory Experiments
}

\author{
${ }^{1,2}$ A. R. Miles, ${ }^{1}$ M. J. Edwards, and ${ }^{1}$ J. A. Greenough \\ ${ }^{1}$ Lawrence Livermore National Laboratory \\ ${ }^{2}$ University of Maryland
}

In core-collapse supernovae, strong blast waves drive interfaces susceptible to Rayleigh-Taylor (RT), Richtmyer-Meshkov (RM), and Kelvin-Helmholtz (KH) instabilities. In addition, perturbation growth can result from material expansion in large-scale velocity gradients behind the shock front. Laser-driven experiments are designed to produce a strongly shocked interface whose evolution is a scaled version of the unstable hydrogen-helium interface in core-collapse supernovae such as SN 1987A. The ultimate goal of this research is to develop an understanding of the effect of hydrodynamic instabilities and the resulting transition to turbulence on supernovae observables that remain as yet unexplained.

In this paper, we summarize recent results from our computational study of unstable systems driven by high Mach number shock and blast waves. For planar multimode systems, compressibility effects preclude the emergence of a regime of self-similar instability growth independent of the initial conditions (IC's) by allowing for memory of the initial conditions to be retained in the mix-width at all times. With higher-dimensional blast waves, divergence restores the properties necessary for establishment of the self-similar state, but achieving it requires very high initial characteristic mode number and high Mach number for the incident blast wave. Initial 
conditions predicted by some recent stellar calculations are incompatible with selfsimilarity.

I. Introduction

The appearance of Supernova 1987A in the Large Magellanic Cloud marked the beginning of a change in the way people think about the violent endpoint of massive stars. Although it had been known for some time that the layered structure of the progenitor should be hydrodynamically unstable during the explosion, ${ }^{1,2}$ the assumption of spherical symmetry was almost always incorporated into models and otherwise reflected in the paradigm of core-collapse supernovae. This was due in large part to the practical limitations of multidimensional numerical calculations. But when heavy elements originating from the core of SN 1987A appeared at the photosphere six months earlier than predicted by one-dimensional explosion models, it became clear that something significant was being neglected. ${ }^{3}$ Since then, evidence that asymmetry is the rule in core-collapse supernovae has continued to accumulate, ${ }^{4,5}$ and multidimensional computer codes have been developed and applied to the problem in an effort to understand proposed asymmetry mechanisms.

One such mechanism is based on the hydrodynamic instability of perturbed interfaces when subjected to a reversal of pressure and density gradients. Due to processes such as convective stirring and localized thermonuclear burn, boundaries between layers of different materials within the star are unlikely to be perfectly 
smooth. Even if the shock wave produced as the core rebounds against neutron degeneracy pressure is initially spherical, it can drive the amplification of any such preexisting perturbations via the Rayleigh-Taylor (RT) ${ }^{11,12}$ and Richtmyer-Meshkov $(\mathrm{RM})^{13,14}$ instabilities. After passage of the shock front, this interface evolves into a complicated structure of outward-growing spikes of heavier material and infalling "bubbles" of lighter elements. ${ }^{7-10}$ Late in time, these spikes can move far ahead of what a $1 \mathrm{D}$ model would predict as the interface position and might explain the anomalously early appearance heavy elements at the photosphere.

In ongoing experiments aimed at studying instability-driven mixing under supernova-relevant conditions, laser energy is used to drive high Mach number planar shock and blast waves into one end of millimeter-scale cylindrical targets. ${ }^{20-28} \mathrm{~A}$ typical target consists of a more dense plastic section and a less dense foam section, with a prescribed perturbation machined into the plastic at the plastic/foam interface. After the passage of the shock, the interface is unstable and evolves under the combined influence of RT, RM, and Kelvin-Helmholtz (KH) instabilities. Additional laser beams directed on high-Z backlighter foils yield $\mathrm{x}$-rays that pass through the target and are used to image the developing instability.

II. Self-similar RT growth and transition to turbulence

For classical RT systems comprised of incompressible fluids under constant acceleration, it is widely believed that memory of the initial conditions is lost at late 
times after the establishment of a self-similar regime. ${ }^{29-31}$ This idea ultimately is based on the simple fact that larger bubbles rise faster than smaller bubbles, and can be explained in terms of bubble competition and merger. ${ }^{32,33}$ As a larger bubble rises above its smaller neighbor, it is free to expand laterally, eventually filling the space previously occupied by its neighbor. Material flowing around the larger bubble and into the spikes below sweeps the smaller bubble downstream. This process leads to the continual generation of larger, faster moving objects and an acceleration of the bubble and spike fronts. Eventually, the interface is dominated by structures resulting from many successive generations of bubble merger rather than from the unstable growth of preexisting perturbations. Loss of memory of the initial conditions means that the statistical properties observed in the late-time interface could have arisen from a wide range of initial perturbation spectra. If the initial conditions are forgotten, the height of the bubble front as well as the dominant transverse scale must grow in proportion to $\mathrm{gt}^{2}$, where $\mathrm{g}$ is the acceleration and $\mathrm{t}$ is time, as this is the only length scale remaining in the problem. The interface can be described in a statistical sense by a bubble-size distribution function. In the self-similar or scale-invariant regime, this function does not change in time except for a scale factor proportional to the characteristic bubble size. Thus any initial distribution must evolve in time towards the scale-invariant distribution.

Although the idea of self-similar RT growth is well motivated, it has yet to be demonstrated conclusively. If the self-similar regime does exist, then it is certainly difficult to reach in simulations and diagnosable experiments. The $\mathrm{gt}^{2}$ scaling is indeed observed, but there is a great deal of disagreement and debate about the 
constant of proportionality $\alpha \cdot{ }^{34}$ Despite limited understanding of nonlinear instability evolution in classical RT systems, the ideas of self-similar growth are sometimes invoked in discussions of blast-wave-driven instabilities in core-collapse supernovae. Even if valid in the classical case, one should question to what extent these ideas would apply in the more complicated blast-wave-driven case, where RM is present, the acceleration is time-dependent, and the flow is compressible.

Whether or not there is a true self-similar regime, hydrodynamically unstable systems certainly can undergo a transition from an early time, more ordered structure to a late time structure that is disordered and appears random. If the Reynolds number is sufficiently high and sufficient time is allotted, this late-time structure will be turbulent.

Many unanswered questions remain, about the requirements for transition and its relationship to loss of memory of initial conditions. In particular, the effect of the initial conditions on the transition is not well understood. In addition, it is also important to understand what effect the transition has on the instability growth rate. For 3D systems, it has been noted that there will be a competition between the continual generation of larger, faster growing structures and the tendency of increased turbulent dissipation to inhibit the growth. ${ }^{35}$ To date, $2 \mathrm{D}$ simulations of mixing in Type II supernovae produce spike velocities about a factor of two smaller than the observed velocities of heavy core elements beyond the photosphere. ${ }^{36}$ It remains unclear whether or not this discrepancy can be resolved once 3D effects, including the transition to turbulence, are included in high-resolution calculations. 
III. Summary of results from models and simulations

We have developed a model that describes the evolution of a blast-wavedriven multimode interface in terms of bubble competition and merger. Our model goes beyond previous work by including the effects of material decompression and stretching behind the shock front for both planar and divergent systems. On the basis of this model, we are able to show that self-similarity and loss of initial conditions might be possible in divergent systems such as supernovae but are not realizable in planar systems such as most laser-driven experiments intended to study mixing in supernovae. The difference arises because modes in divergent systems undergo transverse in addition to radial stretching. Because the time dependence of the stretching is the same in both directions, the ratio of transverse to parallel scales is preserved.

For planar systems, we predict a quasi-self-similar regime during which the instability evolution is approximately self-similar over a limited period of time. During this regime, the ratio of characteristic wavelength to perturbation amplitude decreases slowly in time rather than approaching a constant asymptotic value. Even in the divergent case, loss of initial conditions is possible only for systems with very small-scale initial conditions driven by very high Mach number blast waves. Recent stellar calculations ${ }^{36.40}$ predict significant amplitudes for modes as low as $24-$ 48 due to convection. If these predictions are correct, the late-time interface structure observed in supernova remnants likely depends strongly on the initial conditions present within the star at the time of explosion. 
Finally, the model predicts that the finite duration of the blast-wave drive sets a maximum scale that can be generated on a given interface. For divergent systems, this corresponds to a minimum mode number that depends weakly on the incident Mach number and initial mode number as long as both are sufficiently high (see Fig. 1).

Model predictions for planar systems have been tested against 2D and 3D Raptor $^{38}$ simulations with broadbanded multimode interfaces under NIF-like drive conditions. Raptor uses a higher-order Godunov method and adaptive mesh refinement to solve the compressible Euler equations. The calculation set includes over 70 high-resolution (at least 512 cells in the transverse direction) 2D simulations and five 3D simulations (with 256 cells in each transverse direction). In agreement with our model, the perturbation growth shows no apparent approach to a self-similar regime independent of the initial conditions (see Fig. 2). We also observe the effective box size due to drive decay, which sets a maximum transverse scale that can be generated, and the quasi-self-similar regime. This regime is found to exist after the generation of scales larger than the initial conditions but before the effective box size is reached.

The existence of the quasi-self-similar state and the drive-imposed effective box size make the blast-wave-driven case distinct from classical RT. However, transition to the quasi-self-similar state is very similar to its classical counterpart. In both cases, it is marked by an increase in the degree of small-scale mixing (see Fig. 3), a decrease in the spike velocity, and often an increase in the bubble velocity. 
Significantly, the apparently random variations observed in late-time amplitudes (in Fig. 1) and growth rates are not well correlated with initial spectral shape. Only the average spectral properties are important, such as the initial rms amplitude and characteristic wavenumber. This bodes well for simulations of similarly strongly-driven systems that leave a portion of the short-wavelength end of the spectrum unresolved. As long as the system contains some fast-growing and interacting modes that can be resolved computationally or reproduced experimentally (and has the correct initial rms amplitude), the late-time instability evolution will likely closely resemble the fully resolved or complete system. This reaffirms the hope that laser-driven experiments can serve as useful and relevant platforms for studying compressible mixing in supernovae despite their drastically more limited available range of scales. Similarly, carefully-designed numerical simulations need not necessarily reproduce the full range of spectral details present in their physical counterparts in order to reasonably reproduce the late-time large-scale interface structure. These conclusions apply in particular to systems with long-wavelength modes large enough in amplitude to reach the nonlinear phase early on.

In agreement with 3D classical RT calculations performed by others, we find more fine-scale mixing and small-scale structure in 3D than in 2D after transition to the turbulent-like state (see Fig. 3). The process of transition, however, is very similar in both cases. Transition is triggered by spike interaction and breakdown that is complete when the perturbation amplitude is 5-6 times the characteristic wavelength in the initial spectrum. 
Due to enhanced mixing, the post-transition bubble growth is reduced relative to that observed in the $2 \mathrm{D}$ calculations. The spike growth, however, does not appear to be inhibited and might even be enhanced. This is particularly significant in light of the fact that $2 \mathrm{D}$ supernova calculations that invoke instability-driven mixing to explain enhanced transport of heavy core elements consistently underpredict the latetime spike velocities by about a factor of two.

IV. Future work

We hope to continue this research along several parallel directions directly applicable to both supernovae and ICF applications. We would like to further investigate the effect of spike interactions on their velocity distribution in order to determine the extent to which spike material can be accelerated towards the shock front.

With the greater energy, temporal, and spatial scales afforded by the NIF laser, experiments will potentially be capable of unambiguously demonstrating transition to $3 \mathrm{D}$ turbulence, the generation of larger scales through multiple generations of bubble merger, and the late-time freeze-out stage. Through collaboration with astrophysicists studying supernova progenitors, we will attempt to incorporate realistic initial spectra into the experiments.

A parallel effort would involve the design of planar experiments aimed at studying turbulent mixing in double-shell ignition targets. ${ }^{16}$ One essential aspect of 
these experiments would be the presence of multiple unstable interfaces capable of interacting with one another. Similar interactions are likely important in supernovae, but have typically not been incorporated into the experiments. We expect that the planar double-shell targets can be designed so as to be directly relevant to both systems.

Planar experiments are particularly valuable because of their improved diagnosability relative to spherical systems and their ability to better maintain high energy density in the absence of divergence. However, our buoyancy-drag model has suggested that the absence of divergence changes the nature of the instability evolution by ensuring that memory of the initial conditions is retained in the perturbation amplitudes at all times. Consequently, we are working to develop a divergent platform for supernova-relevant compressible mixing experiments. Finally, we note that current ICF diagnostics in general and x-ray radiography in particular are not optimized for detailed studies of 3D turbulence in laser-drive targets. The long-term success of this program will ultimately depend on the development of innovative new experimental techniques.

\section{$V$. Acknowledgements}

This work was performed under the auspices of the U.S. Department of Energy by the University of California, Lawrence Livermore National Laboratory under Contract W-7405-Eng-48. 
VI. References

${ }^{1}$ S. W. Falk and W. D. Arnett, Astrophys. J. Letters, 180, L65 (1973).

${ }^{2}$ R. Chevalier, Ap. J. 207, 872 (1976).

${ }^{3}$ J. Tueller, S. Barthelmy, N. Gehrels. B. J. Teegarden, M. Leventhal, and C. J.

MacCallum, Astrophys. J. 351, L41 (1990).

${ }^{4}$ L. Wang, D. Baade, P. Höflich, A. Khokhlov, J. C. Wheeler, et al., Astrophys. J.

591, 1110 (2003).

${ }^{5}$ J. P. Hughes, C. E. Rakowski, D. N. Burrows, and P. O. Slane, Astrophys. J. 528, L109 (2000).

${ }^{7}$ W. D. Arnett, J. N. Bachall, R. P. Kirshner, and S. E. Woosley, Ann. Rev. Astron. Astrophys. 27, 629 (1989).

${ }^{8}$ W. Hillebrandt and P. Hoflich, Rep. Prog. Phys. 52, 1421 (1989).

${ }^{9}$ D. Arnett, B. Fryxell, E. Muller, Astrophys. J. 341, L63 (1989); E. Muller, B.

Fryxell, D. Arnett, Astron. Astrophys. 251, 505 (1992)

${ }^{10}$ B. A. Remington, J. Kane, R. P. Drake, et al., Phys. Plasmas 4, 1994 (1997).

${ }^{11}$ J. W. S. Rayleigh, Scientific Papers (University press, Cambridge, 1899).

${ }^{12}$ G. I. Taylor, Proc. R. Soc. London, Ser. A 201, 192 (1950).

${ }^{13}$ R. D. Richtmyer, Commun. Pure Appl. Math. 13, 297 (1960).

${ }^{14}$ E. E. Meshkov, Izv. AN SSSR Mekhanika Zhidkosti I Gaza 4(5), 151 (1969).

${ }^{15}$ John D. Lindl, Inertial Confinement Fusion: the quest for ignition and high-energy 
gain, (Springer-Verlag, New York, 1998).

${ }^{16}$ J. L. Milovich, P. Amendt, M. Marinak, and H. Robey, Phys. Plasmas 11(4), (2004).

${ }^{17}$ J. Hecht, U. Alon, and D. Shvarts, Phys. Fluids 6, 4019 (1994).

${ }^{18}$ U. Alon, J. Hecht, D. Ofer, and D. Shvarts, Phys. Rev. Lett. 74, 534 (1995).

${ }^{19}$ J. M. Soures, R. L. McCrory, C. P. Verdon, et al., Phys. Plasmas 5, 2108 (1996).

${ }^{20}$ K. S. Budil, B. A. Remington, T. A. Peyser, K. O. Mikaelian, P. L. Miller, et al., Phys. Rev. Lett., 76, 4536 (1996).

${ }^{21}$ J. Kane, D. Arnett, B. A. Remington, S. G. Glendinning, J. Castor, et al., Astrophys. J., 478, L75 (1997).

${ }^{22}$ J. Kane, D. Arnett, B. A. Remington, S. G. Glendinning, R. Wallace, et al., in Second Oak Ridge Symposium on Atomic and Nuclear Astrophysics, Oak Ridge, Tenessee, (1998).

${ }^{23}$ J. Kane, D. Arnett, B. A. Remington, S. G. Glendinning, G. Bazan, R. P. Drake, B. A. Fryxell, R. Teyssier, and K. Moore, Phys. Plasmas, 6(5), 2065 (1999).

${ }^{24}$ J. O. Kane, H. F. Robey, B. A. Remington, R. P. Drake, J. Knauer, et al., Phys. Rev. E, 63, 055401R (2001).

${ }^{25}$ H. F. Robey, J. O. Kane, B. A. Remington, R. P. Drake, O. A. Hurricane, et al., Phys. Plasmas, 8, 2446 (2001).

${ }^{26}$ R. P. Drake, H. F. Robey, O. A. Hurricane, Y. Zhang, B. A. Remington, et al., Astrophys. J., 564, 896 (2002).

${ }^{27}$ H. F. Robey, Y. Zhou, A. C. Buckingham, P. Keiter, B. A. Remington, et al., Phys. Plasmas, 10, 614 (2003).

${ }^{28}$ A. R. Miles, D. G. Braun, M. J. Edwards, H. F. Robey, R. P. Drake, et al., 
"Numerical Simulation of Supernova-Relevant Laser-driven Hydro Experiments on OMEGA," to appear in Phys. Plasmas; Chapter 2, this dissertation.

${ }^{29}$ J. Glimm, D. H. Sharp, Phys. Rev. Lett. 64(18), 2137 (1990).

${ }^{30}$ U. Alon, J. Hecht, D. Mulamel, and D. Shvarts, Phys. Rev. Lett. 72(18), 2867

(1994).

${ }^{31}$ D. L. Youngs, Physica D 12, 32 (1984).

${ }^{32}$ K. I. Sharp, Physica D, 12, 3 (1984).

${ }^{33}$ J. Glimm and X. L. Li, Phys. Fluids, 31(8), 2077 (1988).

${ }^{34}$ G. Dimonte and M. Schneider, Phys. Fluids 12(2), 304 (2000).

${ }^{35}$ D. L. Youngs, Laser and Part. Beams 12(4), 725 (1994).

${ }^{36}$ K. Kifonidis, T. Plewa, H.-Th. Janka, and E. Muller, Astron. Astrophys., 408, 621 (2003).

${ }^{37}$ R. T. Barton, Numerical Astrophysics (Jones and Bartlett, Boston, 1985).

${ }^{38}$ L. H. Howell and J.A. Greenough, J. Comp. Phys. 184, 53 (2003).

${ }^{39}$ J. Bell, M. Berger, J. Saltzman, and M. Welcome, SIAM J. Sci. Comput. 15, 127 (1994).

${ }^{40}$ C. A. Meakin and W. D. Arnett, in preparation. 


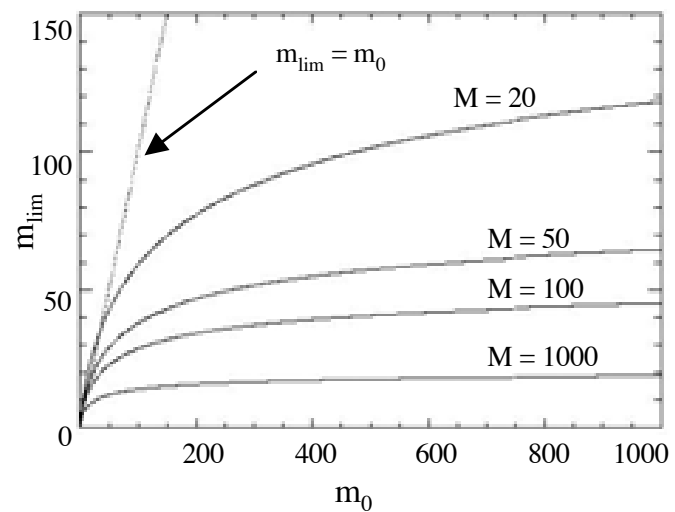

Fig. 1: Model-predicted minimum mode number that can be generated by a spherical blast wave as a function of the initial mode number. Drive decay imposes a limiting mode number for divergent systems. The asymptotic modal structure depends weakly on the initial conditions and the drive strength if the incident shock Mach number and the initial mode number are both is high. 


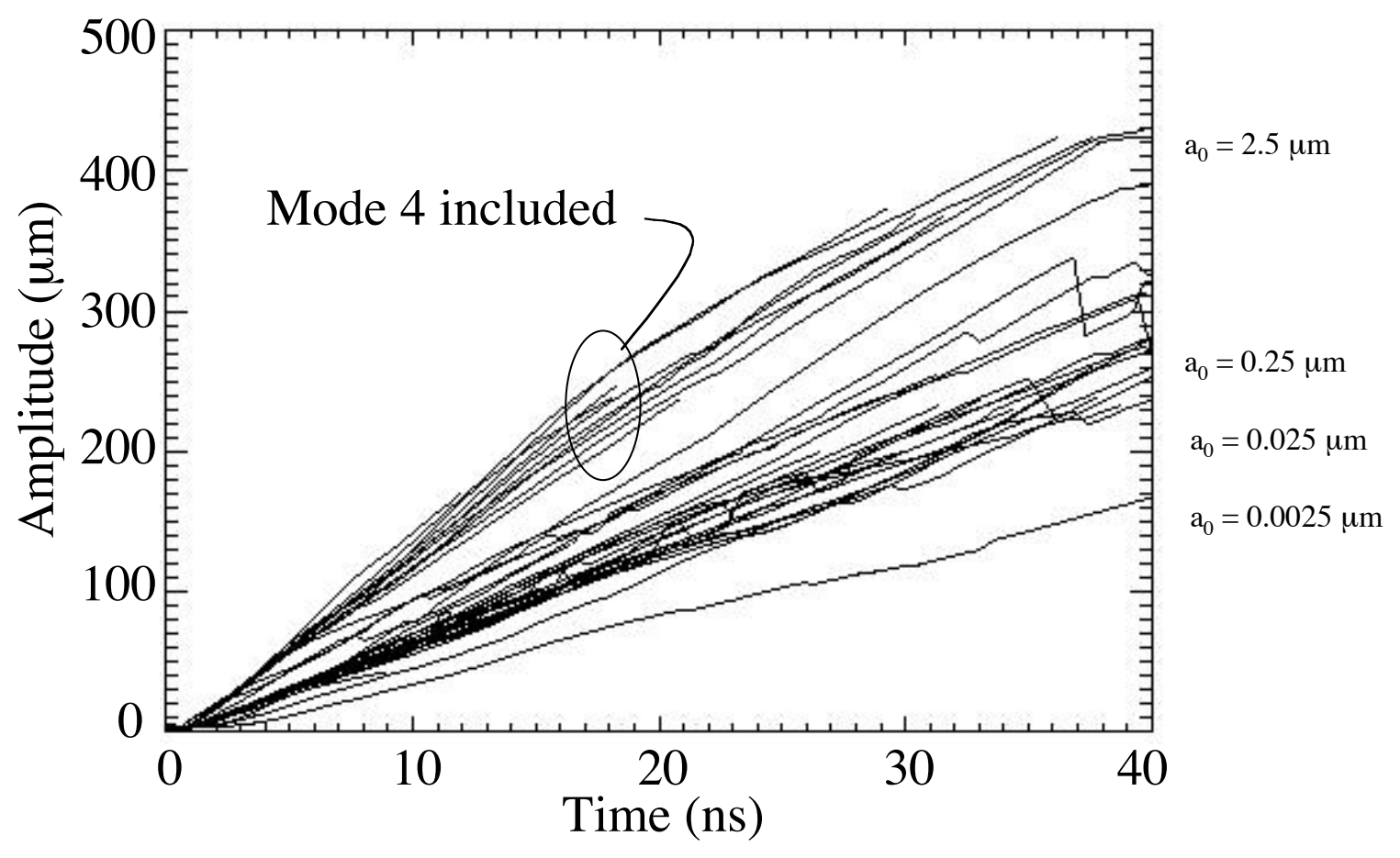

Fig. 2: Amplitude histories from 52 2D simulations of planar laser-driven systems under NIFlike drive conditions. Memory of the initial conditions is retained in the parallel scales at late times. 


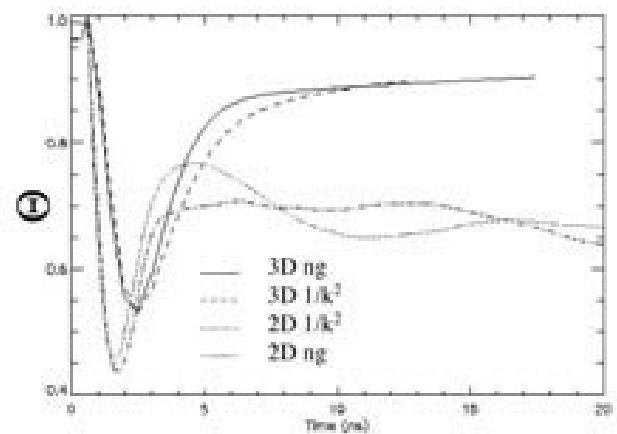

(a)

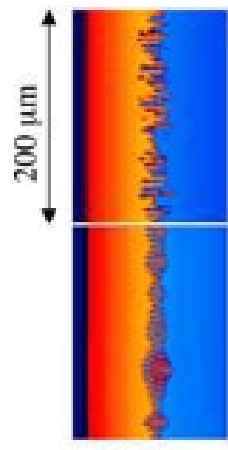

$2.5 \mathrm{~ns}$

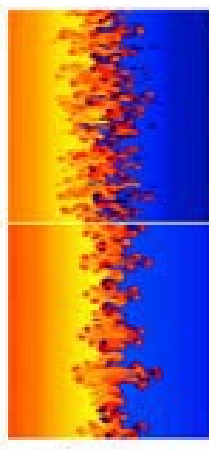

$5.0 \mathrm{~ns}$

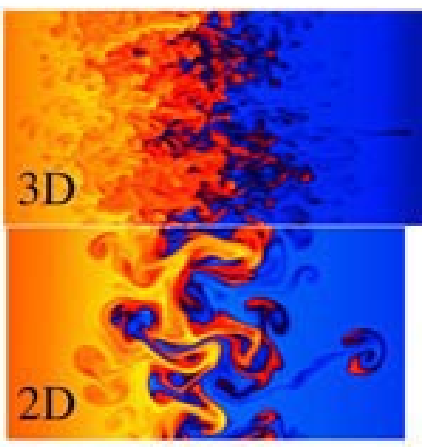

$17.5 \mathrm{~ns}$

(b)

Figure 3: (a) Mixing parameter and (b) log density plots from 2D and 3D calculations with an narrow gaussian initial perturbation spectrum. The mixing parameter is equal to zero when there is no mixing in the interface region and one if the interface material is completely mixed. 